\title{
Point-of-Care for Therapeutic Drug Monitoring of Antineoplastic Drugs
}

\section{Federico Polo* and Giuseppe Toffoli}

Department of Experimental and Clinical Pharmacology, CRO - National Cancer Institute, Aviano, Italy

In the clinical practice, therapeutic drug monitoring (TDM) concerns the analytical determination of pharmaceutical drug concentrations in patients' bio-fluids at specific time intervals to allow a close and timely control of their dosage. This practice allows for rapid medical intervention in case of toxicity-related issues and/or adjustment of dosage to better fit the therapeutic demand.

In the treatment of cancer diseases TDM becomes particularly important, since the antineoplastic drugs show a narrow range of efficacy between non-efficacy and toxicity, which is generally characterized by severe adverse effects.

The therapy is strictly depending on each individual patient, their clinical conditions and characteristics (e.g., weight, age, etc.), and other concomitant drug therapy. Furthermore the pharmacokinetics of any drug is subject to metabolic processes that are finally related to the genomic profile of the patient. Hence, each patient might respond differently to the same therapy. It is then clear that the best results can be obtained when a personalized therapy becomes available.

Clinical medicine has thus to face a challenging task aimed at personalized therapy and real time diagnostics.

However, TDM is currently performed in centralized laboratories equipped with bulky instruments, such as immunoassay analyzers and mass spectrometry, which can be used by trained personnel only. Particularly mass spectrometry, coupled with high-performance liquid chromatography (HPLC-MS), is routinely used in core facilities.

The financial costs related to instrumental operation and maintenance, and the time required for the preparation and analysis of samples, for processing the results, severely affects the application of TDM in medical practices. Therefore, a new generation of analytical tools, capable of providing rapid, sensitive and reliable diagnosis, is necessary to respond to the timely need of drug administration or reduction aiming at effectively treating cancer patients [1].

A device that allows TDM in real-time at point-of-care testing (POCT) $[2,3]$ will help medical doctors avoiding toxic side effects and enhancing drug efficacy [4]. The availability of such devices would allow: (i) to complete analytical tests without involving traditional central laboratories, thus improving the quality and the effectiveness of the process, with a corresponding decrease of costs (benefiting the Health care System); (ii) to improve the effectiveness of a particular anticancer drug, while reducing its toxicity by adjusting the administered therapy in real-time, based on the real plasma concentration, thus leading to an increase of benefits for the patients [4].

Technological advances in the field of nanosciences and biosensors offer the unique opportunity to meet such requirements.

There two essential integrated components that make a sophisticated device to be a biosensor: a biological sensing element (bioreceptor) and a transducer. The former is a biological molecule, such as enzyme, antibody, or aptamer that can specifically interact and recognize the analyte (e.g., biomolecule, synthetic drug) present in the sample. The second component is a system capable of transducing the recognition event into a measurable signal, usually in the form of optical or electrochemical readout. The exquisite blend of sensitivity and specificity of biology and the phyisical properties of transducers offers unique bio-analytical tools that can be easily made portable and user-friendly.

Biosensors can be grouped and classified according to different parameters, but classifying them according to their signal transduction readout and working principles can be helpful, especially when considering that continuous technological novelty can modify and implement such classification. In the past years, a plethora of very interesting reviews dealt with biosensors providing the readers with technological advances and useful applications. We reported some of the most significant and recent ones in the references [5-7]. Essentially, biosensors can be divided into three main types, according to their working principles and detection technology: electrochemical [8], optical [9], and mass-based [10] biosensors.

To date, very few examples are reporting the use of biosensor in TDM application, and almost none are ready for POCT, with the exception of glucose sensor that is the only commercially available device used worldwide, even though it may not be strictly considered a TDM sensor.

However, very recently a real-time biosensor, capable of continuously tracking a wide range of circulating drugs in living subjects, has been developed. The device, based on microfluidic electrochemical detection, was applied for in vivo continuous monitoring (MEDIC). The nanotechnology behind such device rely on electrodes modified with aptamers that are specific for analyte target, doxorubicin (a chemotherapeutic) and kanamycin (an antibiotic). It has been proven to work very nicely with live rats and human whole blood for several hours with high sensitivity and specificity at subminute temporal resolution [11]. MEDIC envisions what it should be expected for the future of therapeutic drug monitoring with real-time measurements using a simple and effective platform.

Another interesting and recent example described a portable device for monitoring an anticancer drug, the folate analog metothrexate (MTX), whose concentration was determined by surface plasmon resonance (SPR). In the assay, folic acid-functionalized gold nanoparticles (FA- AuNP) compete with MTX to bind to human dihydrofolate reductase (hDHFR) immobilized on the SPR sensor chip, thus significantly affecting the signal readout. Promising results were obtained on human serum samples [12].

There are still challenges that the community has to face in the next future to make POCT devices come into reality. Besides the identification of the specific needs required for TDM and exhaustive clinical tests, it is also fundamental that the scientific and medical communities work

${ }^{*}$ Corresponding author: Dr. Federico Polo, Department of Experimental and Clinical Pharmacology, CRO-National Cancer Institute, Via Franco Gallini 2, 33081 Aviano (PN), Italy, Tel: +390434659816; E-mail: federico.polo@cro.it

Received June 02, 2016; Accepted June 03, 2016; Published June 07, 2016

Citation: Polo F, Toffoli G (2016) Point-of-Care for Therapeutic Drug Monitoring of Antineoplastic Drugs. Med chem (Los Angeles) 6: e108. doi:10.4172/21610444.1000e108

Copyright: ( $) 2016$ Polo F, et al. This is an open-access article distributed under the terms of the Creative Commons Attribution License, which permits unrestricted use, distribution, and reproduction in any medium, provided the original author and source are credited. 
Citation: Polo F, Toffoli G (2016) Point-of-Care for Therapeutic Drug Monitoring of Antineoplastic Drugs. Med chem (Los Angeles) 6: e108. doi:10.4172/2161-0444.1000e108

Page 2 of 2

alongside industrial partners to push this technology towards a higher level of development required for regulatory clearance. However, there is very little doubt that, thanks to the advancement in nanoscience and micro/nano-fabrication technologies, biosensors will provide in the very next future new tools for clinical and point-of-care testing, as well as for pharmaceutical drug discovery.

\section{References}

1. National Cancer Institute. Alliance for Nanotechnology in Cancer. Develop Small Tools with a Big Impact on Cancer.

2. Pierce BL, Ballard-Barbash R, Bernstein L, Baumgartner RN, Neuhouser ML, et al. (2009) Elevated Biomarkers of Inflammation are Associated with Reduced Survival among Breast Cancer Patients. J. Clin. Oncol 27: 3437-3444.

3. Kost GJ (1995) Guidelines for point-of-care testing. Improving patient outcomes. Am J Clin Pathol 104: S111-127.

4. Lymberis A (2010) Micro-nano-biosystems: An overview of European research Minim Invasive Ther Allied Technol 19: 136-143.

5. Wang $J$ (1999) Amperometric biosensors for clinical and therapeutic drug monitoring: a review. J Pharm Biomed Anal 19: 47-53.
6. Turner AP (2013) Biosensors: sense and sensibility. Chem Soc Rev 42: 3184-3196.

7. McKeating KS, Aubé A, Masson JF (2016) Biosensors and nanobiosensors for therapeutic drug and response monitoring. Analyst 141: 429-449.

8. Carrara S, Cavallini A, Erokhin V, De Micheli G (2011) Multi-panel drugs detection in human serum for personalized therapy. Biosens Bioelectron 26 : 3914-3919.

9. Griss R, Schena A, Reymond L, Patiny L, Werner D, et al. (2014) Bioluminescent sensor proteins for point-of-care therapeutic drug monitoring. Nat Chem Biol 10: $598-603$.

10. Huang LS, Pheanpanitporn Y, Yen YK, Chang KF, Lin LY, et al. (2014) Detection of the Antiepileptic Drug Phenytoin Using a Single Free-Standing Piezoresistive Microcantilever for Therapeutic Drug Monitoring. Biosens. Bioelectron 59: 233-238.

11. Ferguson BS, Hoggarth DA, Maliniak D, Ploense K, White RJ, et al. (2013) Real-time, aptamer-based tracking of circulating therapeutic agents in living animals. Sci Transl Med 5: 213ra165.

12. Zhao SS, Bukar N, Toulouse JL, Pelechacz D, Robitaille R, et al. (2015) Miniature multi-channel SPR instrument for methotrexate monitoring in clinical samples. Biosens Bioelectron 64: 664-670. 